\title{
TEXTURE IMAGE CLASSIFICATION BY STATISTICAL FEATURES OF WAVELET
}

\author{
Dr. Emmanuel Awuni Kolog \\ Department of Operations and Management Information Systems, \\ University of Ghana, \\ Accra, Ghana \\ eakolog@ug.edu.gh \\ Samuel Nii Odoi Devine \\ Department of Operations and Management Information Systems, \\ Presbyterian University College, \\ Ghana \\ samuel.nodevine@presbyuniversity.edu.gh
}

\begin{abstract}
An unidentified image sample is assigned to a recognized texture class is known as Texture Classification (TC). The main challenging task in TC is the non uniformity changes in orientation, visual appearance and scale. Texture is an important feature in computer analysis for the purpose of classification. In this paper, an efficient TC system based on Discrete Wavelet Transform (DWT) is presented. The performance of the system is evaluated by Brodatz database. At first, the DWT is used to decompose the input texture image for feature extraction at a particular decomposition level. From each sub-band coefficients statistical features are extracted. Finally, k-Nearest Neighbour $(k N N)$ classifier is used for classification. Results show that a better classification accuracy of $94.72 \%$ is achieved by the features of $3^{\text {rd }}$ level DWT and KNN classifier.
\end{abstract}

Keywords: Texture classification, discrete wavelet transform, $k$-nearest neighbor, classification, Brodatz database.

\section{INTRODUCTION}

TC plays an important role for the classification and segmentation of images from different fields. Feature analysis and wavelet based method for texture classification is described in [1]. At first, the wavelet co-occurrence features and statistical features are combined to perform feature extraction. These features are analyzed over different noisy channel like speckle, Poisson, Gaussian and salt and pepper noise. Gabor wavelet features and perceptual texture features based texture image classification is described in [2]. The input texture images are decomposed by Gabor wavelets and DWT. Support Vector Machine (SVM) is used for classification.

A TC system using SVM is presented in [3]. At first, the input texture images are extracted by Gray Level Co-Occurrence Matrix (GLCM), local binary pattern and Gabor wavelet filter. SVM classifier is used for classification. Curvelet transform and SVM based rotation invariant texture classification is described in [4]. Curvelet transform is used to decompose the input texture images. Then, from the subband coefficients of curvelet transform the following features are extracted; circular, isotropic and anisotropic shift. SVM algorithm is used for classification.

Motion statistical feature matrix based dynamic texture classification is presented in [5]. Initially, the input texture images are estimated by the motion vector for dynamic textures and then statistical feature matrix is constructed. 
Finally, the classification is made by two benchmark dynamic textures. Contourlet transform and SVM based texture classification is described in [6]. Contourlet transform is used for the feature extraction of texture images. For the classification of textures, SVM is used.

Wavelet Packet Transform (WPT) based TC is presented in [7] with subset selection and rough set classification. Initially, the input images are decomposed by WPT, and then the features are extracted by L1 normalization. The dominant features are selected by feature subset method. The rough set based classification is used. A color TC system is described in [8] with feature seclection. The Haralick feature is used for feature extraction. The features are selected and classified by non-exhaustive procedure.

Texture classification using local relational string is described in [9]. GLCM is used to extract texture image features. The $k \mathrm{NN}$ classifier is used for the classification. DWT based texture classification is described in [10]. Features are extracted by a patch based approach with different patch sizes from the selected DWT coefficients. The texture image classification is made by Euclidean distance measure.

Dual-tree complex wavelet transform based dynamic texture classification is presented in [11]. Initially, the input texture images are decomposed by dualtree complex wavelet transform. The entropy and variance features are extracted from sub-bands. Then finally, $k \mathrm{NN}$ classifier is used for classification. WPT energy features based texture classification is described in [12]. The input texture images are extracted by WPT. From, the wavelet packet coefficients energy features are extracted. The Mahalanobis distance classifier is used for the classification.

An efficient method for TC using DWT and KNN classifier is presented in this paper. The organization of the paper is as follows: The methods and materials used for TC system are discussed in section 2. Section 3 discusses about the results and discussion obtained by the DWT and KNN classifier for TC system. The last section concludes the TC system employed on Brodatz textures.

\section{METHODS AND MATERIALS}

The overall block diagram for texture image classification is shown in Figure 1. This system has two stages; feature extraction and classification. These stages are explained in following sections.

\section{A. DWT based feature extraction}

The discretely sampled wavelets in functional and numerical analysis are known as DWT. DWT is also used in other fields like electrocardiogram signal classification [13] and image watermarking [14]. Comparing to other wavelet transforms DWT captures location information and frequency and also it has Fourier transform in temporal resolution. The translation scales and some rules of discrete set of wavelets are employed in DWT. The DWT function is defined by,

$$
\delta(z)=\sum_{k=-\infty}^{\infty}(-1)^{k} d_{N-1-k} \delta(2 z-k)
$$

where $N$ is an even integer. The input texture images are decomposed by DWT and it produces the low and high frequency sub-band coefficients. From these sub-band coefficients, statistical features of DWT such as mean, standard deviation, variance, skewness and kurtosis are extracted. 
Int. J.Adv.Sig.Img.Sci, Vol. 5, No.1, 2019

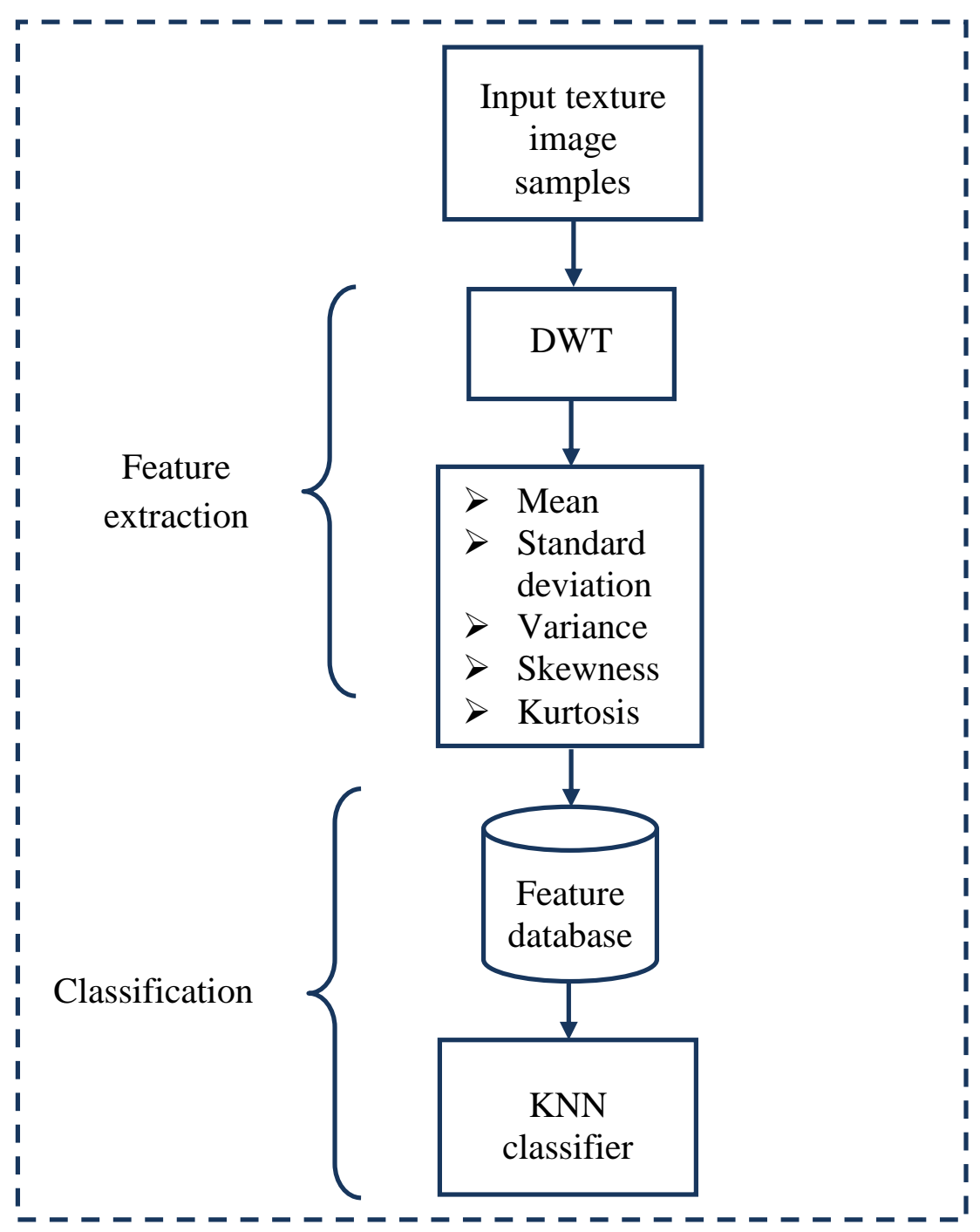

Fig. 1 Block diagram of TC system by DWT and kNN

\section{B. kNN Classification}

KNN is a non parametric method used for regression and classification. In KNN algorithm the input consist of $k$ closest neighbors in the feature space. KNN helps to store the available classes based on measures of data. The KNN rule is assigned by the $k$ neighbors. The $k$ represents the number of pixels from the neighbor such as, $k=1,2,3, \ldots, n$ here $n$ is the number of cases. The KNN is measured by Euclidean distance. Consider $g=\left(e_{1}, f_{1}\right)$ and $h=\left(e_{2}, f_{2}\right)$ are two points. Euclidean distance between these two points is given by,

$$
(g, h)=\sqrt{\left(e_{1}-e_{2}\right)^{2}+\left(f_{1}-f_{2}\right)^{2}}
$$

For the $n$-dimension such as $g=\left(e_{1}, e_{2}, e_{3}, \ldots e_{n}\right)$ and $h=\left(f_{1}, f_{2}, f_{3}, \ldots f_{n}\right)$ then the Euclidean distance between these two points is given by, 


$$
(g, h)=\sqrt{\left(e_{1}-f_{1}\right)^{2}+\left(e_{2}-f_{2}\right)^{2}+\ldots+\left(e_{n}-f_{n}\right)^{2}}
$$

In this study, the $k \mathrm{NN}$ classifier is used for the classification of textures. The $k \mathrm{NN}$ classifier is used in other fields like under water object recognition [15] and paddy disease recognition [16].

\section{RESULTS AND DISCUSSION}

In this section, the performances of $\mathrm{TC}$ system on Brodatz texture are discussed. The database consists of 25 texture images.
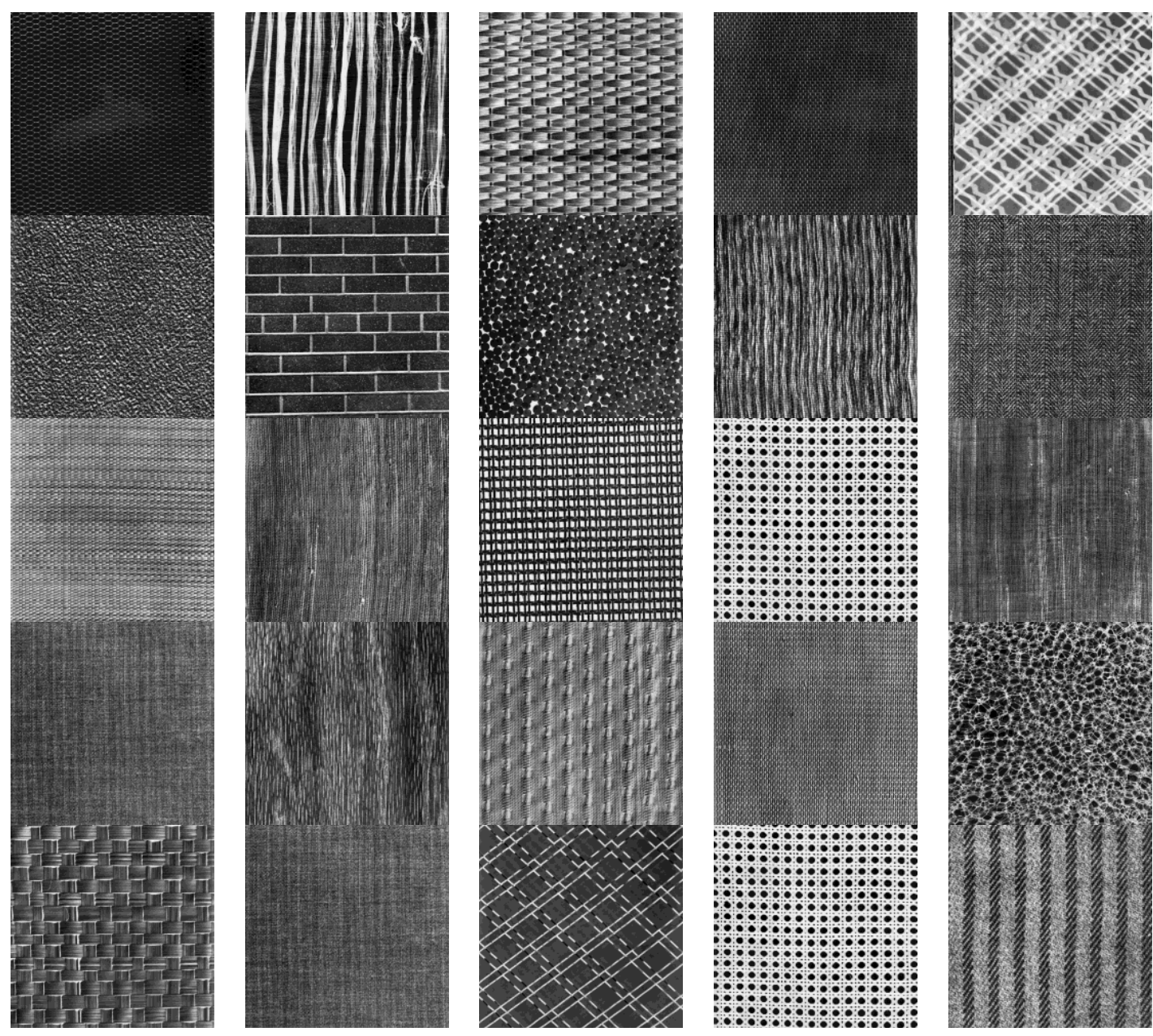

Fig. 2 Brodatz database - sample texture images

The size of images in the Brodatz texture database is 640x640 pixels with 256 gray levels. From the original image, size of $128 \times 128$ image patches are extracted by overlapping 32 pixels between horizontal and vertical direction. This process creates 256 image patches per texture image. Among them, 40 image patches per texture are randomly selected for training and the remaining image patches per texture images are used for testing. 
At first, the input texture images are decomposed by DWT at $3^{\text {rd }}$ level of decomposition. It produces one (1) low frequent sub-band and nine (9) higher frequency sub-bands. Figure 3 shows a $3^{\text {rd }}$ level DWT decomposition of a texture image in Brodatz database.

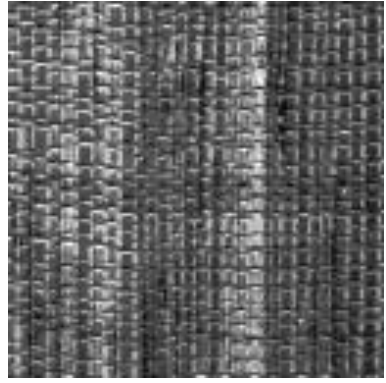

(a)

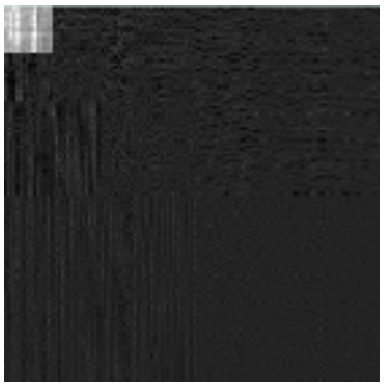

(b)

Fig. 3 DWT decomposition of Brodatz image (a) Original Image (b) DWT at $3^{\text {rd }}$ level

From the ten (10) sub-bands, statistical features are extracted. These extracted features are given to $k N N$ classifier. Table 1 shows the classification accuracy of texture images with their texture ID.

TABLE 1 Classification accuracy of texture images based on DWT and kNN classifier

\begin{tabular}{|c|c|c|c|c|c|}
\hline $\begin{array}{c}\text { Texture } \\
\text { ID }\end{array}$ & $\begin{array}{c}\text { Classification } \\
\text { Accuracy (\%) }\end{array}$ & $\begin{array}{c}\text { Texture } \\
\text { ID }\end{array}$ & $\begin{array}{c}\text { Classification } \\
\text { Accuracy (\%) }\end{array}$ & $\begin{array}{c}\text { Texture } \\
\text { ID }\end{array}$ & $\begin{array}{c}\text { Classification } \\
\text { Accuracy (\%) }\end{array}$ \\
\hline D6 & 92 & D47 & 97 & D76 & 100 \\
\hline D11 & 94 & D51 & 96 & D78 & 96 \\
\hline D16 & 96 & D53 & 93 & D79 & 93 \\
\hline D17 & 94 & D55 & 93 & D83 & 94 \\
\hline D20 & 96 & D56 & 95 & D101 & 91 \\
\hline D21 & 93 & D57 & 94 & D102 & 96 \\
\hline D26 & 96 & D64 & 96 & D111 & 95 \\
\hline D34 & 93 & D66 & 95 & Average & 94.72 \\
\hline D46 & 95 & D68 & 95 & & \\
\hline
\end{tabular}

It is observed from Table 1 , that the overall classification accuracy obtained by 25 texture images is $94.72 \%$ by using the DWT based KNN classifier. Among the 25 texture images only 13 images produces higher accuracies above $95 \%$ and only texture D76 produces the $100 \%$ accuracy by $3^{\text {rd }}$ level DWT features and KNN classifier.

\section{CONCLUSION}

In this study, an efficient TC system based on DWT with the help of $k \mathrm{NN}$ classifier is presented. At first, DWT is used to represent texture into different resolutions that produce the lower and higher frequency sub-band coefficients. Then the mean, standard deviation, variance, skewness and kurtosis features are 
extracted as statistical features from the DWT sub-bands. These statistical features are the inputs for $k N N$ classification. The performance of the system on Brodatz database images provides promising results. Experimental results show the better classification accuracy of $94.72 \%$ while using $3^{\text {rd }}$ level DWT features and $k \mathrm{NN}$ classifier.

\section{REFERENCES}

[1]. M.S. Zaid, R.J. Jadhav, and P.J. Deore, "An efficient wavelet based approach for texture classification with feature analysis", IEEE International Advance Computing Conference, 2013, pp. 1149-1153.

[2]. M. Jian, L. Liu, and F. Guo, "Texture image classification using perceptual texture features and Gabor wavelet features", Asia-Pacific Conference on Information Processing, Vol. 2, 2009, pp. 55-58.

[3]. Y.B. Salem, and S. Nasri, "Rotation invariant texture classification using support vector machines", International Conference on Communications, Computing and Control Applications, 2011, pp. 1-6.

[4]. Y. Shang, Y.H. Diao, and C.M. Li, "Rotation invariant texture classification algorithm based on curvelet transform and SVM", International Conference on Machine Learning and Cybernetics, Vol. 5, 2008, pp. 3032-3036.

[5]. Y. Qiao, Y. Zhao, and X. Song, "Dynamic Texture Classification Based on Motion Statistical Feature Matrix", International Conference on Intelligent Information Hiding and Multimedia Signal Processing, 2013, pp. 535-538.

[6]. Z. Xiangbin, "Texture classification based on contourlet and support vector machines", International Colloquium on Computing, Communication, Control, and Management, Vol. 2, 2009, pp. 521-524.

[7]. Q. Wang, H. Li, and J. Liu, "Subset selection using rough set in wavelet packet based texture classification", International Conference on Wavelet Analysis and Pattern Recognition, Vol. 2, 2008, pp. 662-666.

[8]. A. Porebski, N. Vandenbroucke, and L. Macaire, "Iterative feature selection for color texture classification", IEEE International Conference on Image Processing, Vol. 3, 2007, pp. III-509.

[9]. A. Hafiane, and B. Zavidovique, "Local relational string for textures classification", International Conference on Image Processing, 2006, pp. 2157-2160.

[10]. A. Jayasudha, and D. Pugazhenthi, "Classification of Textures using Patch Based Energy Features of Selected Wavelet Coefficients", Journal of Applied Sciences, Vol. 14, No. 7, 2014, pp. 709-713.

[11]. Q. Yu-long, and W. Fu-shan, "Dynamic texture classification based on dual-tree complex wavelet transform", International Conference on Instrumentation, Measurement, Computer, Communication and Control, 2011, pp. 823-826.

[12]. M.C. Lee, and C.M. Pun, "Texture classification using dominant wavelet packet energy features", IEEE Southwest Symposium on Image Analysis and Interpretation, 2000, pp. 301-304.

[13]. S. Pattnaik, M. Dash, and S.K. Sabut, "DWT-based feature extraction and classification for motor imaginary EEG signals", International Conference on Systems in Medicine and Biology, 2016, pp. 186-201.

[14]. A. Senol, K. Dınçer, H. Sever, and E. Elbaşi, "Blocked-DWT based vector image watermarking", Signal Processing and Communications Applications Conference, 2015, pp. 264-267. 
[15]. S. Murugan, and C. Srinivasan, "Underwater Object Recognition Using KNN Classifier", International Journal of MC Square Scientific Research Vol.9, No.3, 2017, pp. 48-52.

[16]. M. Suresha, K.N. Shreekanth, and B.V. Thirumalesh, "Recognition of diseases in paddy leaves using knn classifier", International Conference for Convergence in Technology, 2017, pp. 663-666. 